\title{
LAS CORRIENTES PEDAGÓGICAS Y LOS DESAFÍOS DE LA EDUCACIÓN
}

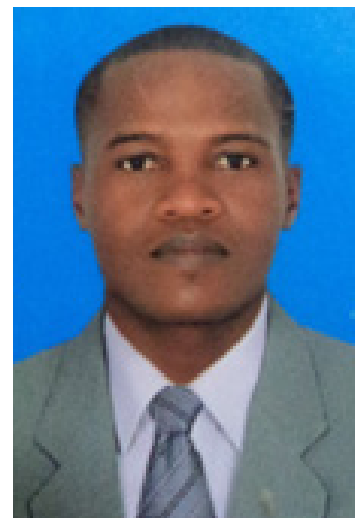

Fecha de recepción: 01/09/2017

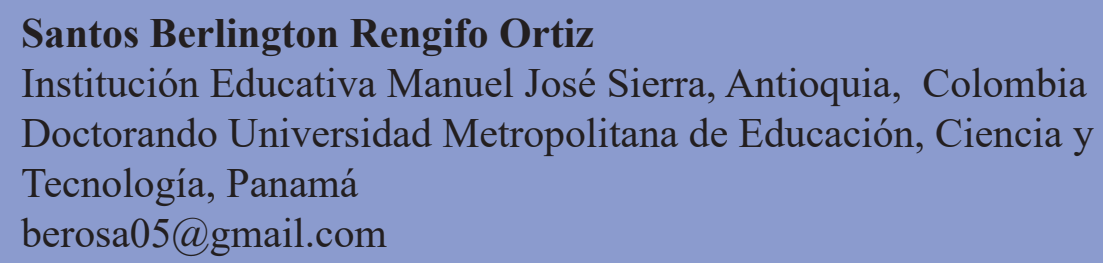

\section{RESUMEN}

El presente ensayo aborda la educación, en un recorrido que se realiza desde diferentes periodos de la historia de la educación, comenzando desde el siglo de las luces, pasando por la edad de oro, hasta llegar a la educación actual, en esta última se enfatizan diferentes tipos de pedagogías: las emergentes, la de la esperanza y la planetaria, además se realiza un análisis de los filósofos y pedagogos que más se han destacado en cada una de esas épocas resaltando en cada uno de ellos: que finalidad tiene frente a la educación, cuál es su concepto frente al niño y frente al maestro y el método que ha desarrollo en función de la educación de los niños independientemente de su contexto. Posteriormente se plantean unas conclusiones que recogen los elementos más sobresaliente de las épocas ya mencionadas, en función de dejar evidencias de una mirada puntual frente los actores del proceso educativo; finalmente se plantea unos desafíos que apuntan a una educación más moderna y pensada en el niño como eje central del proceso, y quienes son el motor de la educación, para llegar a los maestros los cuales son más vistos como guías y orientadores de los diferentes proceso de enseñanza y aprendizaje, que el niño desarrolla en un contexto especifico. Además, se puede evidenciar las referencias bibliográficas las cuales recogen los autores citados en el documento y que fueron determinantes para el desarrollo del presente escrito.

Palabras clave: Educación, niño, maestro, método y enseñanza. 


\title{
THE PEDAGOGICAL CURRENTS AND THE CHALLENGES OF EDUCATION
}

\begin{abstract}
This essay deals with education, in a journey that takes place from different periods of the history of education, starting from the century of lights, through the golden age, until reaching the current education, in the latter they are emphasized different types of pedagogies: the emergent ones, the one of the hope and the planetary one, in addition an analysis of the philosophers and pedagogues was realized who have stood out in each one of those times that they emphasize in each one of them: that the intention has in front of education, which is their concept in front of the child and in front of the teacher and the method that has development according to the education of the children regardless of their context. Afterwards, some conclusions are presented that include the most outstanding elements of the times and nowadays, in order to leave evidence of a punctual look before the actors of the educational process; Finally, there are challenges that point to a more modern education and thought of the child as the central axis of the process, and who are the engine of education, to reach teachers, who are seen more as guides and counselors of the different processes .Of teaching and learning, that the child develops in a specific context. In addition, you can see the bibliographic references which include the authors cited in the document and which were decisive for the development of this writing.
\end{abstract}

Keywords: Education, child, teacher, method and teaching.

\section{A MANERA DE INTRODUCCIÓN}

La educación está enmarcada en tres momentos dominantes, (el siglo de las luces, la edad de oro y la educación actual) que han permitido a la educación dejar una radiografía de cómo la evolución en la historia, permite conocer tanto sus ventajas como desventajas y dejar en evidencia los postulados de los autores en relación con: los fines de la educación, las ideas del niño, el rol de maestro, sus métodos y formas como visionan los contenidos, en diferentes épocas y momento de la historia.

El primer momento es el siglo de las luces, etapa que está comprendida desde la antigüedad hasta el siglo XVIII, los pensadores que han marcado esta época han desarrollado diferentes teorías que permiten reflexionar sobre los problemas del conocimiento, del saber, y del ser como parte fundamental. A continuación, se relacionan diferentes filósofos que se han destacado y han dejado, un legado para la humanidad como base para el desarrollo de diferentes procesos.

El padre de la didáctica Juan Amos Comenio, el iniciador de las ilustraciones en los libros y 
el teatro en los niños, proclama el lema "las escuela práctica, sin malos tratos" en el análisis que hace (Gadotti, 2011), se plantea una educación que interprete y prolongue la experiencia de cada día y la utilización de los medios clásicos, como la enseñanza de la religión y de la ética, en la práctica de la enseñanza, fue el pionero en la aplicación de métodos que despertaran el creciente interés de los educandos.

Para Comenio, la educación debe ser comprensiva, no memorística, que haga parte de un proceso para toda la vida, que integre las actividades creativas, humanas y sus principios para una amplia reforma social basada en la unión de la teoría y la práctica.

Tanto para Comenio, como para Robín Paul uno de los pioneros de la educación social libertaria, se debe educar al niño de una forma integral, en la que se tenga en cuenta como centro del proceso, que sean seres que encarnen la paz, en la práctica recomienda eliminar los castigos en las aulas. Que los educandos comprendan lo que hacen y lo hagan de una forma consiente y agradable.

El maestro según Comenio debía conocer primero las cosas que enseñaba. Además, debe aprender que no debe avanzar mientras los conocimientos básicos no estén firmes en la mente del alumno, La docencia para Comenio era el oficio más noble entre todos.

Comenio en relación con la enseñanza, expresa tres elementos: el tiempo, el objeto y el método. Este último presentado como imitación de la naturaleza, debe ser único, dejando de evidencia el enseñar - aprender, y el leer - escribir; lo cual se ve de una forma más amplia en su obra la didáctica magna en la cual recomienda que para aprender y enseñar se debe proceder de lo conocido a lo desconocido.

En relación con el método pedagógico, tiene como base los procesos naturales del aprendizaje: la inducción, la observación, los sentidos y la razón. Parte de su propuesta pedagógica era eliminar totalmente la violencia del proceso educativo además exigió con firmeza que la educación primaria fuera obligatoria.

Otro de los grandes pedagogos de la historia es Pestalozzi, quien luchaba por una reforma de la sociedad a través de la educación de las clases populares. Él mismo se puso al servicio de sus ideas creando un instituto para niños huérfanos, fundó el famoso Internado de Yverdon que fue frecuentado durante sus veinte años de funcionamiento por estudiantes de todos los países de Europa donde impartía una educación en contacto con el ambiente inmediato, siguiendo una objetiva, progresiva y gradualmente un método natural y armonioso.

Para Pestalozzi, el fin de la educación es formar seres humanos íntegros. Esto comprende el aspecto cognitivo, respetando las etapas evolutivas y la individualidad; el aspecto social, moral y 
físico; entrenarlos para el mundo laboral, por lo cual en el currículum debían incluirse actividades agrícolas. (Fingernarr, 2010).

El aprendizaje del niño según Pestalozzi, debe ser un proceso "que simplemente sigue el camino de la naturaleza, que guie al niño lentamente a través de su propio esfuerzo. (Morrison, 2004, pág. 62), se apoya en algunos conceptos de Rousseau, de una forma especial en el uso de la naturaleza como parte del aprendizaje.

Para Pestalozzi los maestros deben estar bien preparados para lograr un desarrollo integral del alumno más que para implantarles conocimientos. Sus ideas ejercieron gran influencia en las escuelas del mundo occidental, particularmente en el área de preparación de los maestros (León, 1997).

Pestalozzi planeta un método experimental en el que los niños puedan: observar, medir, dibujar, escribir y así en enseñar a distinguir la forma de cada objeto. Se enseña a describir o a darse cuenta de sus percepciones, siempre a partir de las cosas simples a lo más difícil. Promulgó la importancia del juego en los procesos pedagógicos del niño, de una forma orientada.

Según Rousseau, el fin de la educación es formar al hombre natural. Enseñarle a vivir, enseñarle a enfrentar la vida con libertad. (Quiroz, 2011), una buena educación debe estar ligada a la naturaleza del niño y a su contexto que permita que él viva el aprendizaje como algo propio de su cotidianidad, esta situación permitirá una mayor apropiación de los conocimientos.

Para Rousseau, el niño vive en un mundo propio que es necesario comprender y es en este punto donde los docentes deben sacar su ingenio y lograr entender ese mundo que permita potenciar esos aprendizajes, y lograr en ellos potenciar sus saberes.

El maestro deberá, pues, hacerse cargo del deseo del niño dejándolo libre y hasta obligándole a serlo. El respeto de esta segunda exigencia se podrá garantizar mediante un proyecto pedagógico claro y preciso en el que la instrucción se hará por la "necesidad de las cosas", fuera de alcance de la voluntad humana (Soëtard, 1999).

Rousseau, plantea un método natural para una educación integral, en el que lo fundamental era el uso de la razón, con respecto a este en su tesis pedagógica plantea: La naturaleza fija las etapas del educando, la acción natural satisface el interés del momento.

Para Kant la educación debe desarrollar en el individuo la capacidad de servirse por sí mismo desde su propio entendimiento. Este tipo de educación pensado en la capacidad de servicio, de dar y aportar a los demás; apunta al trabajo colaborativo de una forma compartida, que permita que el conocimiento se fortalezca entre los pares. 
Para Kant el educando se debe cultivar y civilizarse, para así corresponder a la naturaleza. De esa forma, el verdadero objetivo del hombre es que desarrolle completamente, por sí mismo (Gadotti, 2011). Este pensamiento de Kant es muy importante, ya que invita a los formadores a plantear diferentes acciones que permitan que el niño desarrolle sus potencialidades y las ponga al servicio de la su realidad y cotidianidad.

Kant hace la diferencia entre dos tipos de maestros: el instructor que es el que educa para la escuela y el ayo ${ }^{1}$ quien es el encargado de educar para la vida. El maestro debe de enseñar al niño a pensar y a elegir sólo lo bueno alejándolo de lo malo de lo perjudicial.

Utiliza la experiencia como un camino para llegar al aprendizaje y lograr que en esa relación el individuo pueda alcanzar un nuevo conocimiento. Las escuelas deben ser públicas y además se menciona a los institutos de educación cuyo fin es el perfeccionar la educación doméstica. Se busca una educación experimental, ya que la educación no debe ser sólo mecánica sino razonada.

Los contenidos y temáticas educativas están ligadas a las relaciones de los maestros con sus educandos y la importancia de contar con una buena empatía entre ellos (Manacorda, 2007) lo expresa de la siguiente manera.

La tradición oral le dio contenido simbólico a todo el quehacer de las comunidades y grupos humanos. Así se aseguró el aprendizaje y la continuidad de los grupos, hasta miles de años después, que se inventaron la escritura. Este invento produjo la primera gran revolución en la educación. Los textos escritos comenzaron a vincular buena parte del conocimiento, y produjeron cambios también en las cosas que aprendía la gente; nacieron así las primeras escuelas. En ellas, predominó el modelo transmisionista; el profesor tenía unos conocimientos que dictaba y hacía repetir a los alumnos, quienes a su vez los aprendían, para más tarde enseñarlos de la misma manera (Manacorda, 2007).

Los contenidos de la educación consisten en cuerpos de información y de habilidades que se elaboraron en el pasado; la tarea principal de la escuela es, por consiguiente, transmitirlos a la nueva generación. En el pasado, patrones y reglas de conducta se establecieron; en consecuencia, la educación moral consiste en adquirir hábitos de acción en conformidad con tales reglas y patrones.

La segunda etapa también denominada momento pedagógico o edad de oro (1880-1970), la cual ingresa la pedagogía al ámbito de la educación, permitiendo de esta manera que la teoría y la práctica siempre estén ligadas en el mismo escenario independientemente del contexto y de los medios en el que se esté inmerso; en este sentido (Carvajal, 2017) expresa:

${ }^{1}$ f. Persona encargada en las casas principales de custodiar niños o jóvenes y de cuidar de su crianza y educación. Real Academia Española (C) Todos los derechos reservados 
Las grandes instituciones pedagógicas que ponen al estudiante en el centro del proceso educativo; que valorizan la experiencia como componente fundamental del aprendizaje; que enfatizan procesos de socialización y educación para la libertad; que hablan de educación integral o del impacto político de la educación que nos vamos a permitir relacionar autores y pensamientos cuya afinidad es en definitiva la innovación pedagógica y el deseo de formar bien a los jóvenes y asegurar el progreso de la sociedad.

En esta etapa la educación pasa a ser manejada por el estado y sujeta a la política de un territorio determinado, enfatizando en los valores y en la ética como principios primordiales.

El pedagogo Pablo Robín propone una educación integral, racional y mixta, impulsando talleres que formen a los jóvenes en oficios útiles. (Robin, 1908), es importante en la finalidad de Robín, ya que el educado no se puede pude mirar solo por un aspecto, sino de una forma conjunta y desde todos sus ángulos, es decir de una forma integral.

Robín Paul plantea una teoría de la educación integral, como el camino para la superación de la alineación del hombre ante al sistema capitalista de exploración, además se buscan las condiciones para que cada persona descubra sus propias habilidades basadas en las inclinaciones de cada uno. Para él un maestro debe plantear un trabajo práctico ligado a la observación, para llegar a la teoría, de una forma conjunta y consiente.

Froebel, plantea que, al niño, se le debe educar desde tres líneas: el juego, las manualidades y el estudio de la naturaleza, los tres elementos son importante y muy necesario en los procesos de enseñanza aprendizaje, en este sentido es importante retomar que el niño aprende de una forma diferente y esto es una buena estrategia para aplicar.

Ferrer por su aparte expresa una educación con libertad de pensamiento y acción, para enfrentar la nueva era. Es importante tener el tacto necesario para identificar hasta qué punto llega el tipo de libertad que se propone en esta generación, en este sentido se requiere de cierto grado de autonomía que en su conjunto serían muy difíciles de manejar y controlar en un establecimiento educativo determinado.

Ferrer fue un revolucionario que creía en el valor de la educación, como remedio absoluto para los males de la sociedad. Se consideraba un profesor que amaba a los niños y quería prepararlos, con libertad de pensamiento y acción, para enfrentar una nueva era. Plantea una educación que aplique un método lúdico, que tenga el juego como herramienta principal de desarrollo, eliminando así de la escuela el aburrimiento y la falta de atención de los infantes, actualmente Ferrer es reconocido como uno de los pioneros de la escuela moderna. 
Antón Semionovich Makarenko (1888-1939) expresa que la educación tiene como objeto lograr una persona autónoma moralmente, capaz de crear su propio sistema de valores y ajustar su conducta a él. Es importante que la educación recupere la práctica de los valores en las aulas y fuera de ella ya que es algo que realmente se está perdiendo día tras día.

Makarenko, también sufrió la influencia del movimiento de la Escuela Nueva, propuso la escuela única hasta los diez años, fundamentada en la "autoridad de la ayuda", para él ser educador es una cuestión de personalidad y carácter - capacidades innatas y no de teoría, estudio y aprendizaje.

Para Dewey la educación debe ser un proceso y no un producto, un proceso de reconstrucción y reconstitución de la experiencia. En este fin se reconoce la importancia de una enseñanza articula y secuencial que permita que los niños articulen los conceptos y que estos a su vez vayan generando conocimiento.

Planteo una escuela basada en lo social, en la que se desarrolle un espíritu de cooperación social y de vida comunitaria, en la que la vida sea el complemento de todos y esto pueda generar una vida de común, un trabajo en conjunto donde todos nos beneficiemos.

Dewey rechaza un conjunto de doctrinas pedagógicas de variado significado; la educación como preparación, como desenvolvimiento, como adiestramiento de la facultad o como formación; se pretende con esto que la escuela sea un espacio de aprendizaje para todos, donde el conocimiento se construya de una forma conjunta.

Pare él "el niño aprende de su experiencia directa con la realidad, aplica la metodología aprender haciendo" (Espot, 2006) en la que el niño se involucre en el aprendizaje y de esta manera participe de ella, logrando una mayor motivación para desarrollar las diferentes actividades programadas. En función del maestro "requiere que los maestros realicen una tarea extremadamente difícil, que es reincorporar a los temas de estudio en la experiencia" (Westbrook, 1999)

Dewey plantea un "método experimental" el cual se basa en la educación de la destreza individual, de la iniciativa y del espíritu de empresa. Sus escritos influyeron en los profundos cambios experimentados en la pedagogía de Estados Unidos en los inicios del siglo XX." (Red de profesionales de la educación, 2014)

Montessori por su parte, plantea que los ambientes espaciales deben estar dispuestos para que las experiencias de aprendizaje de los niños sean mejores. Esto permitirá que los educados tengan una mayor apropiación de los contenidos que se están desarrollando en el aula.

Montessori expresa que "cada niño es único, tiene su personalidad propia, su ritmo de vida, sus 
cualidades y sus posibles dificultades" (Gillles, 2017), en relación con los espacio que relacionan a los niños, estos están cargado de una cantidad de recursos didácticos que facilitan el aprendizaje y el desarrollo motriz de los educandos.

Montessori conseguía hacer que los niños, por el tacto, por la presión, pudieran distinguir los colores, las formas de los objetos, los espacios, los ruidos, la solidez, etc. Su método desarrolla una pedagogía de la libertad, la cual se caracteriza por proveer un ambiente preparado: ordenado, estético, simple, real, donde cada elemento tiene su razón de ser en el desarrollo de los niños. El aula Montessoriana integra edades agrupadas en períodos de 3 años, lo que promueve naturalmente la socialización, el respeto y la solidaridad.

La educación en esta etapa (la edad de oro), en términos generales está limitada en que los educandos adquirieran los elementos básicos para defenderse, pero con una alta mirada a la formación del ser. Para los niños era importante leer, escribir, hacer cuentas, se sigue utilizando las plantillas para reproducir el modelo trazado por el maestro, el contenido de la enseñanza estaba organizado desde las artes liberales y todo lo relacionado con la doctrina cristiana.

Las artes liberales son agrupadas y definidas como Trívium por un lado (gramática, dialéctica y retórica) y el quadrivium por el otro (aritmética, geometría, astronomía y música).

El aprendizaje de contenidos y recursos didácticos, se iniciaba con difícil y laborioso aprendizaje de la lectura y la escritura a muy temprana edad y duraba muchos años, después se proseguía con la gramática y el estudio de los textos clásicos y religiosos, y culminaba con un conjunto de disciplinas de carácter más especializado: leyes, reglamentos y enseñanzas técnicas basadas en conocimientos matemáticos. El método básicamente estaba enfocado en la copia o dictado, memorización y recitado.

Las instituciones educativas comprendían primero el entorno familiar, con la iniciación de los niños en las costumbres familiares, sociales y ético-religiosas y probablemente también era iniciado en algún tipo de profesión que requiere especialización como la agricultura o la artesanía. La instrucción propiamente dicha, especialmente la lectura y la escritura, y la adquisición de conocimientos científicos se realizaba en la escuela en dos niveles: el elemental y el superior.

La tercera etapa, es la que vivimos en la última cuarta década, en la que la educación coge un carácter científico y se ha inscrito en el marco de la ideología dominante caracterizada por el carácter científico.

${ }^{2}$ Designaba los estudios que tenían como propósito ofrecer conocimientos generales y destrezas intelectuales científico-lingüísticas. 
En las pedagogías emergentes (Jordi, 2013) se plantean dos temas importantes, el primero sobre la integración de las TIC (Tecnologías de la Información y la Comunicación) en la labor educativa y en segundo lugar, analizó las desventajas o las anti-practicas pedagógicas en el uso de las tecnologías en las aulas.

Este tipo de pedagogías, posee una visión de la educación que va más allá de la adquisición de conocimientos o de habilidades concretas. Educar es también ofrecer oportunidades para que tengan lugar cambios significativos en la manera de entender y actuar en el mundo.

Los docentes y los aprendices asumen riesgos intelectuales y transitan por caminos nuevos y que estimulan el pensamiento, con actividades creativas, divergentes y abiertas, no mera repetición, los niños por su aparte hay que educarlos para que creen su futuro, los profesores tienen que trabajar para que a los alumnos les guste ir al colegio y tengan otra visión de escuela. Para lograr tal fin es importante que se conviertan las actividades escolares en experiencias personales significativas y auténticas, que estimulen el compromiso emocional de los participantes. Jordi, hace un llamado a los profesores para que fomenten una educación moderna, aprovechando lo que nos ofrecen las TIC, pero siempre con sabiduría crítica y sin renunciar al lado más natural de la enseñanza.

El doctor (Pilonieta, 2016) por su parte, plantea una educación de la esperanza, representada en la modificabilidad estructural cognitiva, la cual tiene como principio, que el organismo humano es un sistema abierto, que en su evolución, adquirió la propensión para modificarse a sí mismo, siempre y cuando se genere un acto humano mediador.

En este sentido, los profesores son los llamados a mediar en el proceso de los educandos con los niños, percibiéndolos individualmente, distinguiéndolos de los otros, deben desarrollar en ellos la apreciación de sus valores propios, sin olvidar los de los demás, ni sus diferencias psicológicas.

La búsqueda del conocimiento no se encasilla y necesita del error y del riesgo para que pueda surgir la reflexión $\mathrm{y}$, por medio de ésta, se transciendan los saberes determinantes.

En este mismo sentido es importante traer a la escena el texto de (Morin, Roger, \& Motta, 2002) en la cual los autores entiende a esta nueva era como aquella que trata de comprender la condición humana, para lo cual, se debe adquirir sentido aventurero y de gran apertura, pero, sobre todo, poder rebuscar e indagar en la historia de la evolución humana las posibles respuestas para el cambio y la reestructuración de las políticas vigentes.

Educar en la era planetaria apunta a formar bajo un pensamiento complejo, la cual permite formas diferentes de ver las cosas, como tal vez existan muchas más, pero lo que busca es llegar a eso, la era planetaria, esa correlación del hombre con el mundo, de sentirnos humanos, de llevar el buen vivir como filosofía propia y trabajar por vivir bien en el presente y construir el futuro de nuestros semejantes. 
Ver la educación desde un mundo globalizado, es vincular al sistema varios tipos de competencias, que se puedan evidenciar en su contexto o en la sociedad en la cual se esté inmerso, esta es una de las razones por las cuales el tipo de pregunta y respuesta ha venido variando en los exámenes de Estado. Ya no basta la memoria o la claridad del concepto, se hace necesario saber poner en contexto los contenidos, los aprendizajes.

Según (Mejia, 2001) "El nuevo conocimiento es de base tecnológica y comienza a darse el conocimiento multi-pragmático, es decir un trabajador multiusos que sepa de todo un poco apoyando así el nuevo sistema de producción dando forma al trabajador flexible del capitalismo globalizado" esta una radiografía de la realizada actual que viven los países latino americanos en los cuales, es importantes tener un buen portafolios de conocimientos que permitan adquirir deferentes empleos en fusión de sobre vivir.

Actualmente la educación, vista desde la globalización no es un panorama muy prometedor, los sistemas educativos están visto desde los lineamientos de las empresas capitalistas, las políticas de turno o las orientaciones de diferentes organismos como la OCDE, FMI, OMC, BM o países dominantes, que son el producto de la pérdida de autonomía de los países en vía de desarrollo.

Analizada las tres etapas de la filosofía de la educación es importante plantear diferentes conclusiones, en función de dejar de evidencia una mirada puntual frente los actores del proceso educativo.

La educación está llena de muchas visiones, pensamientos y acciones que han permitido una trasformación en las universidades, en los colegios, en las aulas de clases, y los seres como tal, hoy parece que todos estos elementos solo fueron cosa del pasado, la gran tarea de los trabajadores de la educación es repensar la práctica y retomar el rumbo.

Los educandos en las diferentes experiencias expuestas, logran avances, sumado a diferentes actividades como: participación activa, protagonismo, vinculación en los procesos, aportes por medios de las experiencias, espacio agradables y enriquecidos de muchos elementos, entre otros.

Los pedagogos y filósofos, de la época plantearon un docente, con capacidad para forma el niño bajo el manejo de diferentes visiones: tener en cuenta los procesos y los tiempos, entenderlo y conocer sus necesidades, enseñarles a pensar y otorgarles la libertad para hacerlo.

Los método o caminos para llegar al conocimiento permiten una mayor asimilación de sus contenidos, algunos de los ya expuestos están relacionados con: usar la naturaleza como laboratorio para el aprendizaje, que la experimentación haga parte de la práctica, aplicar la libertad como parte del aula que permita al educando ser el mismo, aprender haciendo con el conocimiento y que este se convierta en creador del futuro y trasformados de su cotidianidad. 
Para finiquitar el presente texto se requiere de desafíos que apunten a una educación más moderna y pensada para toda una población que día a día requiere de una mayor atención y cuidado.

La educación actual está en un proceso de transición muy complicado (tiempo líquido) y difícil de manejar, hay muchos caminos posibles las tecnologías de la información y de la comunicación y los contextos sociales, son elementos que han tomado mucha fuerza y que hacen parte de las aulas y del proceso de enseñanza aprendizaje, como un elemento más, fortaleciéndolos y simplemente dificultado su desarrollo. Situación que obliga a la comunidad educativa a repensar la práctica.

Se requiere de una educación más pensada en el ser y en sus valores que permita recuperar su identidad y tener una mayor claridad frente su presente y su futuro inmediato.

El sistema educativo requiere de una reestructuración y con ella los métodos, contenidos, objetivos y modelos de aprendizajes, pero que además el contexto este en el centro del escenario de la discusión, con elemento indispensable para un mejor desarrollo.

Es necesario retomar el dialogo en el aula de una forma organizada y direccionada, que permita que el educando exprese sus saberes y sus dudas frente a una situación específica.

Se debe ofrecer una educación que vincule el estímulo, la comprensión, la creativa humana, la orientación y el acompañamiento en los diferentes procesos que se requieren.

Los maestros en la educación moderna requieren reinventarse a favor de los niños y niñas, su papel ya es de mediador y guía u orientador del proceso, pero además debe ser una persona muy creativa que permita descubrir en cada niño sus potenciales y conocimientos.

Retomar la formación en valores, la moral y la ética, ya que hay una ausencia en el respeto al otro y su dignidad, hay mayor desintegración de las familias, los niños cada día están más desprotegidos, los padres están más dedicados al trabajo, a la actualidad y sus intereses personales, la falta de oportunidades y las pocas políticas sociales de los gobernantes de turno y muchos otros elementos, han aportado a la desintegración de esta sociedad.

Desarrollar una educación pensada en el ser, que permita que sea agente principal de sus procesos y se desarrolle como parte de sus necesidades y no como el aparato administrativo que la dirige, además que esté ligada a la actualidad como elemento agregado.

Volver a estructurar esos espacios agradables para los educandos, con decoraciones acorde a las edades, con sentido pedagógico y formativo, pero a la vez decorativo, que exprese un ambiente agradable y digno para la educación y la comunidad en general. 
Plantear ejercicios experimentales, usando el aula y sus espacios como un laboratorio de aprendizaje, con el desarrollo de diferentes actividades de ciencia, matemáticas, español, artes, pintura, canto, danza, música entre otros; que permitan a los educados desarrollar otro tipo de habilidades que quizás no se pueden explorar en el desarrollo de las actividades convencionales del aula.

\section{Referencias Bibliografía}

Carvajal, J. (2017). Corrientes pedagógica y filosóficas de la educación.

Espot, M. (2006). La autoridad del profesor . Recuperado el 07 de 10 de 2017, de https:// books.google.com.co/books?id=XmO1L0QMucMC\&pg=PA81\&dq=que+es+el+ni\%C3\%B $10+\% 2 B+$ para + dewey\&hl=es\&sa $=X \& v e d=0$ ahUKEwj-t9LwjODWAhUE6CYKHSM0BV

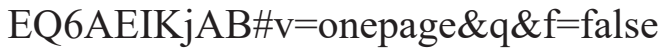

Fingernarr, H. (27 de 10 de 2010). Recuperado el 05 de 10 de 2017, de https://educacion. laguia2000.com/general/pestalozzi-y-la-educacion

Gadotti, M. (2011). Historia de las ideas pedagógicas. Mexico: Siglo XXI.

Gillles, D. (2017). Montesori en casa . Recuperado el 07 de 10 de 2017, de https://books. google.com.co/books?id=y7MiDgAAQBAJ\&printsec=frontcover\&dq=que + es + el + ni $\% \mathrm{C}$ $3 \% \mathrm{~B} 1 \mathrm{o}+\% 2 \mathrm{~B}+$ para + montessori\&hl $=\mathrm{es} \& \mathrm{sa}=\mathrm{X} \& \mathrm{ved}=0 \mathrm{ahUKEwj} 3 \times 8 \mathrm{vjjuDWAhVISiYKH}$ XDLADgQ6AEINzAD\#v=onepage \&q=que $\% 20$ es $\% 20 \mathrm{el} \% 20 \mathrm{ni} \% \mathrm{C} 3 \% \mathrm{~B} 1 \mathrm{o} \% 20 \% 2 \mathrm{~B} \% 20$ para\% 20 montessori\& $\mathrm{f}=$ false

Jordi, A. (2013). La pedagogía del siglo XXI - 2 - Pegagogías emergentes y tecnologías emergentes. Recuperado el 10 de 10 de 2017, de La pedagogía del siglo XXI - 2 - Pegagogías emergentes y tecnologías emergentes

León, I. M. (1997). Principios y técnicas para la elaboracion de material didáctico. Recuperado el 07 de 10 de 2017, de https://books.google.com.co/books?id=gRkbZDIqdvcC\&pg=PA20 $\& \mathrm{dq}=\mathrm{el}+$ papel + del + maestro + segun + pestalozzi $\& \mathrm{hl}=\mathrm{es} \& \mathrm{sa}=\mathrm{X} \& \mathrm{ved}=0$ ahUKEwizrKCY-OH WAhUFOSYKHboUDekQ6AEIJTAA\# $\mathrm{v}=$ onepage $\& \mathrm{q}=\mathrm{el} \% 20$ papel $\% 20 \mathrm{del} \% 20$ maestro $\% 20$ segun $\% 20$ pestalozzi\&f $=$ false

Manacorda, M. A. (2007). Historia de la Educación 1, de la antiguedad hasta 1500. México: Siglo XXI Editores. 
Mejia, R. (2001). Educacion(es) en la(s) Gloalizacion(es). Bogota: Desde abajo.

Morin, E., Roger , E., \& Motta , R. (2002). Educar en la era planetaria. Barcelona: Gedisa.

Morrison, G. (2004). Educacion Infantil . Recuperado el 07 de 10 de 2017, de https://books. google.com.co/books?id=BBJWBEQTARAC\&pg=PA62\&dq=que + concepto + tiene + pest

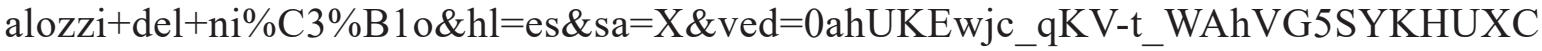
ALQQ6AEIJTAA\#v=onepage $\& q=$ que $\% 20$ concepto $\% 20$ tiene $\% 20$ pestalozzi $\% 20 \mathrm{del} \% 20$ ni $\% \mathrm{C} 3 \% \mathrm{~B} 1$ o\&f $=$ false

Pilonieta, G. (2016). Entrevista del Doctor José Carvajal . Recuperado el 10 de 10 de 2017 , de Modificabilidad estructural Cognitiva.: https://www.youtube.com/watch?v=YAzeibsx61E

Quiroz, E. R. (08 de 2011). Teoria de la Educacion . Recuperado el 05 de 10 de 2017, de http://peducativas.blogspot.com.co/2011/09/fines-de-la-educacion-segun-juan-jacobo.html

Red de profesionales de la educación. (2014). Jhon Dewey. Recuperado el 07 de 10 de 2017, de http://pedagogia.mx/john-dewey/

Robin, P. (1908). Biografia . Recuperado el 6 de 10 de 2017, de http://www.filosofia.org/ aut/001/1908paul.htm

Soëtard, M. (1999). JEAN-JACQUES ROUSSEAU. Paris: Unesco.

Westbrook, R. (1999). JOHN DEWEY. Paris : Unesco. 